\title{
Patterns of Synchrony in Coupled Cell Networks with Multiple Arrows*
}

\author{
Martin Golubitsky ${ }^{\dagger}$, lan Stewart ${ }^{\ddagger}$, and Andrei Török ${ }^{\dagger}$
}

\begin{abstract}
A coupled cell system is a network of dynamical systems, or "cells," coupled together. The architecture of a coupled cell network is a graph that indicates how cells are coupled and which cells are equivalent. Stewart, Golubitsky, and Pivato presented a framework for coupled cell systems that permits a classification of robust synchrony in terms of network architecture. They also studied the existence of other robust dynamical patterns using a concept of quotient network. There are two difficulties with their approach. First, there are examples of networks with robust patterns of synchrony that are not included in their class of networks; and second, vector fields on the quotient do not in general lift to vector fields on the original network, thus complicating genericity arguments. We enlarge the class of coupled systems under consideration by allowing two cells to be coupled in more than one way, and we show that this approach resolves both difficulties. The theory that we develop, the "multiarrow formalism," parallels that of Stewart, Golubitsky, and Pivato. In addition, we prove that the pattern of synchrony generated by a hyperbolic equilibrium is rigid (the pattern does not change under small admissible perturbations) if and only if the pattern corresponds to a balanced equivalence relation. Finally, we use quotient networks to discuss Hopf bifurcation in homogeneous cell systems with two-color balanced equivalence relations.
\end{abstract}

Key words. coupled systems, synchrony, bifurcation

AMS subject classifications. 34C15, 34C23, 34A34, 37C99, 37G15

DOI. $10.1137 / 040612634$

1. Introduction. Stewart, Golubitsky, and Pivato [10] formalize the definition of a coupled cell system in terms of the symmetry groupoid of an associated coupled cell network and prove three general theorems about such networks. First, a set of cells can be robustly synchronous if and only if the cells are in the same equivalence class of some balanced equivalence relation. Second, every balanced relation leads to a new coupled cell network, called a quotient network, that is formed by identifying equivalent cells. Third, the restriction of a coupled cell system to a synchrony subspace (or polydiagonal) is a coupled cell system associated to the quotient network. The approach in [10] has two difficulties:

(1) Not every coupled cell system of ODEs corresponding to the quotient network is the restriction of a coupled cell system corresponding to the original network. This fact makes it difficult to prove genericity statements about dynamics in the original network based only on genericity statements about dynamics of the quotient network. (Dias and

\footnotetext{
${ }^{*}$ Received by the editors October 21, 2003; accepted for publication (in revised form) by G. Kriegsmann July 30 , 2004; published electronically February 22, 2005. This work was supported in part by NSF grant DMS-0244529 and ARP grant 003652-0032-2001.

http://www.siam.org/journals/siads/4-1/61263.html

${ }^{\dagger}$ Department of Mathematics, University of Houston, Houston, TX 77204-3008 (mg@math.uh.edu, torok@math.uh.edu).

${ }^{\ddagger}$ Mathematics Institute, University of Warwick, Coventry CV4 7AL, UK (ins@maths.warwick.ac.uk). The work of this author was supported in part by a grant from EPSRC.
} 
Stewart [3] obtain necessary and sufficient conditions, on a network with a balanced relation, for every quotient system to be a restriction of a cell system corresponding to the original network.)

(2) Reasonable networks that are not included in the theory developed in [10] can exhibit patterns of robust synchrony. Examples are linear chains with Neumann boundary conditions considered in Epstein and Golubitsky [5] and square arrays of cells with Neumann boundary conditions considered in Gillis and Golubitsky [6].

In this paper we show that both of these difficulties can be resolved if the class of coupled cell networks is enlarged to permit multiple couplings between cells and self-coupling. We call this the multiarrow formalism for coupled cell networks. Although the abstract definition of this enlarged class of coupled cell networks is more complicated than the more restrictive definition in [10], the multiarrow formalism has the side benefit that quotient systems are more easily defined in the enlarged class and have more convenient properties.

We first motivate the generalization by considering two examples in the important case of a homogeneous network, which we now define. A cell is a system of ODEs, and a coupled cell system is a collection of $N$ cells with couplings. As discussed in [10], a class of coupled cell systems is defined by a coupled cell network, which is a (directed, labeled) graph that specifies, among other information, which cells are coupled to which. Two cells of the network are input isomorphic (see [10]) if the dynamics of the cells are specified by the same differential equations, up to a permutation of the variables. More precisely, if cells 1 and 2 with internal state variables $x_{1}, x_{2} \in \mathbf{R}^{k}$ are input isomorphic, then the relevant components of the system of ODEs take the form

$$
\begin{aligned}
& \dot{x}_{1}=f\left(x_{1}, y_{1}, \ldots, y_{l}\right), \\
& \dot{x}_{2}=f\left(x_{2}, z_{1}, \ldots, z_{l}\right),
\end{aligned}
$$

where the $y_{j}$ (resp., $z_{j}$ ) are internal state variables of the cells connected to cell 1 (resp., cell 2). In particular, the two cells receive inputs from the same number $l$ of cells, the input variables are of the same type $y_{j}, z_{j} \in \mathbf{R}^{k_{j}}$, and the dependence of the corresponding components of $\dot{x}$ is specified using the same function $f$ of the relevant internal variables and input variables. The phase space of the coupled cell system is

$$
P=\left\{x=\left(x_{1}, \ldots, x_{n}\right) \in \mathbf{R}^{k_{1}} \times \cdots \times \mathbf{R}^{k_{N}}\right\} .
$$

We call a coupled cell network homogeneous if all cells are input isomorphic (in which case $k_{1}=\cdots=k_{N}$ ). Homogeneous coupled cell systems are determined by a single function $f$, as illustrated in (1.1). For the remainder of this introduction we focus on homogeneous coupled cell networks.

We can visualize an equivalence relation $\bowtie$ on cells by coloring all equivalent cells with the same color. This equivalence relation is balanced (in the homogeneous case with one kind of coupling) if the sets of colors of input cells for two equivalent cells consist of the same colors with the same multiplicities. Theorem 6.5 of [10] states that the subspace

$$
\Delta_{\bowtie}=\left\{x \in P: x_{i}=x_{j} \text { if } i \bowtie j\right\}
$$

is flow-invariant for all $f$ if and only if $\bowtie$ is balanced. A solution in $\Delta_{\bowtie}$ is synchronous in the strong sense that the time series from cells of the same color are identical; the synchrony is 
robust in the sense that it holds for any choice of $f$. We call $\Delta_{\bowtie}$ the polydiagonal or synchrony subspace corresponding to $\bowtie$.

Quotients lead to multiple arrows. We describe circumstances in which multiple arrows are natural and useful. Consider the homogeneous five-cell coupled cell network pictured in Figure 1 (left). A balanced coloring of this network is given in the right panel of that figure.
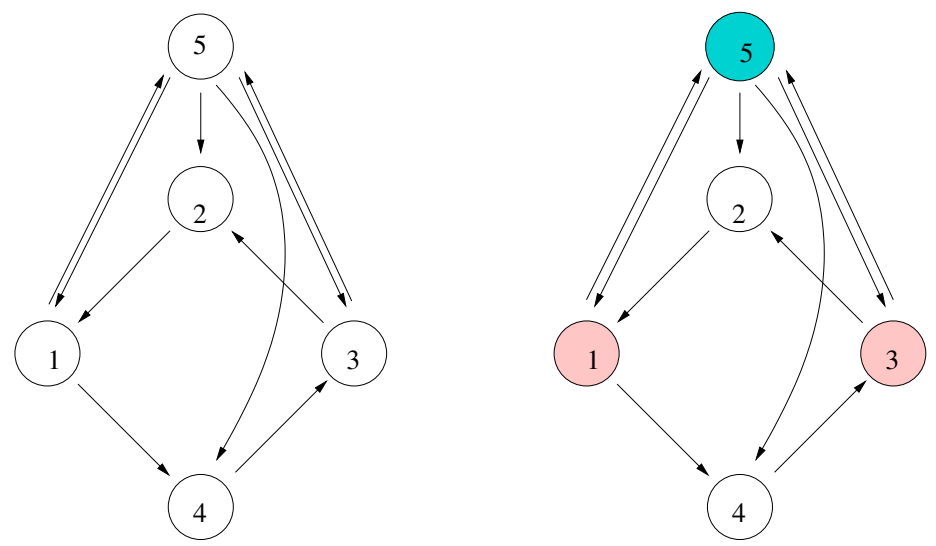

Figure 1. (Left) Homogeneous five-cell network. (Right) Balanced coloring of the network.

The differential equations corresponding to this five-cell network have the form

$$
\begin{aligned}
& \dot{x}_{1}=f\left(x_{1}, x_{2}, x_{5}\right), \\
& \dot{x}_{2}=f\left(x_{2}, x_{3}, x_{5}\right), \\
& \dot{x}_{3}=f\left(x_{3}, x_{4}, x_{5}\right), \\
& \dot{x}_{4}=f\left(x_{4}, x_{1}, x_{5}\right), \\
& \dot{x}_{5}=f\left(x_{5}, x_{1}, x_{3}\right),
\end{aligned}
$$

where $f(a, b, c)=f(a, c, b)$ since all couplings are assumed to be identical. It is straightforward to check that the subspace $\Delta$ defined by $x_{1}=x_{3}$ and $x_{2}=x_{4}$ is flow-invariant. The restricted system on $\Delta$ has the form

$$
\begin{aligned}
& \dot{x}_{1}=f\left(x_{1}, x_{2}, x_{5}\right), \\
& \dot{x}_{2}=f\left(x_{2}, x_{1}, x_{5}\right), \\
& \dot{x}_{5}=f\left(x_{5}, x_{1}, x_{1}\right) .
\end{aligned}
$$

The quotient cell construction in [10] leads to the coupled cell network of Figure 2 (left). The coupled cell system corresponding to that quotient network, which is not homogeneous, has the form

$$
\begin{aligned}
\dot{w} & =f(w, p, c), \\
\dot{p} & =f(p, w, c), \\
\dot{c} & =g(c, p) .
\end{aligned}
$$

Therefore, a coupled cell system corresponding to the quotient network is the restriction of a coupled cell system corresponding to the five-cell network if and only if $g(b, w)=f(b, w, w)$, 
where $f(a, b, c)=f(a, c, b)$. In this paper we remove such conditions from consideration by allowing multiple couplings between cells. With multiple couplings, the quotient network is the homogeneous one of Figure 2 (right). Quotient coupled cell systems for the new quotient have the form

$$
\begin{aligned}
\dot{w} & =f(w, p, c), \\
\dot{p} & =f(p, w, c), \\
\dot{c} & =f(c, p, p),
\end{aligned}
$$

and each of these systems is the restriction to $\Delta$ of a five-cell system. Homogeneous three-cell networks with each cell having at most two input arrows are classified in [9]. See Figure 8; there are 34 such networks.
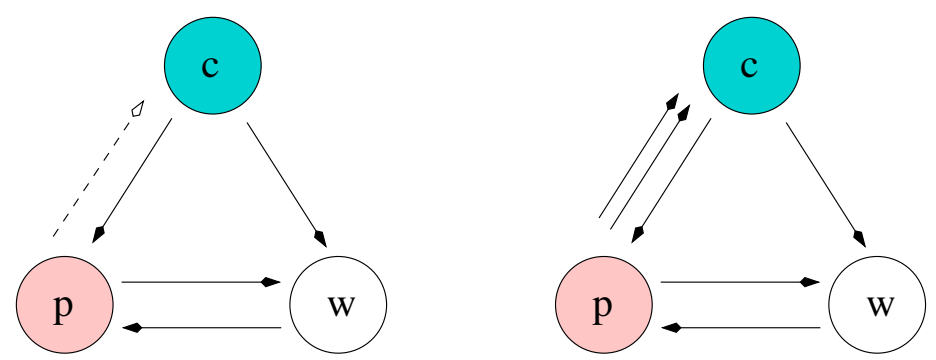

Figure 2. (Left) Three-cell quotient from [10] of five-cell network in Figure 1 (right). (Right) Three-cell quotient using multiarrows.

Neumann boundary conditions lead to self-coupling. We now provide a reason for permitting self-coupling. Epstein and Golubitsky [5] consider patterns of synchrony in $N$-cell bidirectional linear arrays with Neumann boundary conditions. The systems of ODEs have the form

$$
\begin{aligned}
\dot{x}_{1} & =f\left(x_{1}, x_{1}, x_{2}\right), \\
\dot{x}_{j} & =f\left(x_{j}, x_{j-1}, x_{j+1}\right), \quad 1<j<N, \\
\dot{x}_{N} & =f\left(x_{N}, x_{N-1}, x_{N}\right),
\end{aligned}
$$

where $f(a, b, c)=f(a, c, b)$. When self-coupling of a cell to itself is allowed, the network architecture is the one pictured in Figure 3.

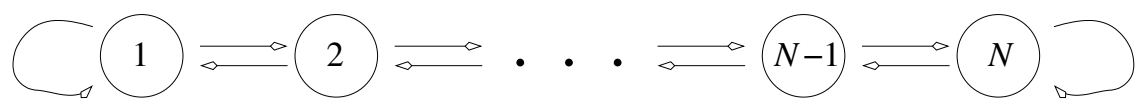

Figure 3. Linear array network.

The ten-cell array in Figure 4 provides an example of a balanced coloring that cannot be obtained from the results in [10], since self-coupling is not permitted in that theory. To see that this coloring is balanced observe that each pink cell (cells $1,5,6,10$ ) receives an input from one pink cell and one white cell, each cyan cell (cells 3,8$)$ receives inputs from two white cells, and each white cell (cells $2,4,7,9$ ) receives inputs from one pink cell and one cyan cell. See also [7]. 


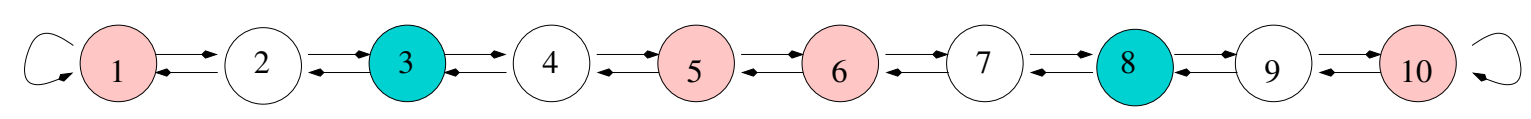

Figure 4. Linear array network of ten cells with a three-color balanced relation.

Structure of the paper. The paper is structured as follows. The enlarged class of "multiarrow" coupled cell networks, which permits multiple arrows and self-coupling, is defined in section 2. The associated admissible vector fields are constructed in section 3 . In that section we also show that distinct networks in the enlarged class can correspond to the same space of admissible vector fields. We call two such networks ODE-equivalent. This (undesired) feature is not present in the class of networks considered in [10]. The connection between balanced equivalence relations and robust polysynchrony is discussed in section 4 . In this section we prove Theorem 4.3, which states that flow-invariant subspaces correspond to balanced equivalence relations in the multiarrow formalism. Quotient networks are defined in the context of multiple arrows and self-coupling in section 5. In Theorem 5.2 we show that all admissible vector fields on a quotient network lift to admissible vector fields on the original network, a property that fails for the quotients defined in [10].

Some examples of coupled cell networks with self-coupling and multiple arrows are discussed in section 6 . The important special case of identical-edge homogeneous networks (homogeneous networks in which all coupling arrows are equivalent) is considered in section 8. Proposition 8.2 states that every homogeneous network with multiarrows and/or self-coupling is a quotient of a homogeneous network with neither multiarrows nor self-coupling. In symmetric networks, Hopf bifurcation typically leads to periodic states in which some cells have identical waveforms (hence identical amplitudes) except for a well-defined phase shift. In section 9 we show that in identical-edge homogeneous networks, Hopf bifurcation can lead to periodic states with well-defined approximate phase shifts and different amplitudes.

As noted, Theorem 4.3 states that flow-invariant subspaces can be identified with balanced equivalence relations. Theorem 7.6 strengthens this result by showing that if a hyperbolic equilibrium has a pattern of synchrony that does not change under small admissible perturbations, then the subspace corresponding to this pattern of synchrony is flow-invariant and hence corresponds to a balanced equivalence relation.

The proofs of several of the main theorems in this paper (particularly Theorems 4.3 and $5.2(\mathrm{a}))$ are straightforward adaptations of corresponding results in [10] to the enlarged category of networks considered here. The general results that go beyond those in [10] are the lifting of quotient vector fields (Theorem 5.2(b)) and the result that rigid hyperbolic equilibria correspond to balanced equivalence relations (Theorem 7.6).

2. Coupled cell networks. We begin by formally defining a class of coupled cell networks that permits multiple arrows and self-couplings.

Definition 2.1. In the multiarrow formalism, a coupled cell network $\mathcal{G}$ consists of the following:

(a) A finite set $\mathcal{C}=\{1, \ldots, N\}$ of nodes or cells.

(b) An equivalence relation $\sim_{C}$ on cells in $\mathcal{C}$. The type or cell label of cell $c$ is the $\sim_{C^{-}}$ equivalence class $[c]_{C}$ of $c$. 
(c) A finite set $\mathcal{E}$ of edges or arrows.

(d) An equivalence relation $\sim_{E}$ on edges in $\mathcal{E}$. The type or coupling label of edge $e$ is the $\sim_{E}$-equivalence class $[e]_{E}$ of $e$.

(e) Two maps $\mathcal{H}: \mathcal{E} \rightarrow \mathcal{C}$ and $\mathcal{T}: \mathcal{E} \rightarrow \mathcal{C}$. For $e \in \mathcal{E}$ we call $\mathcal{H}(e)$ the head of $e$ and $\mathcal{T}(e)$ the tail of $e$.

We also require a consistency condition:

(f) Equivalent arrows have equivalent tails and heads. That is, if $e_{1}, e_{2} \in \mathcal{E}$ and $e_{1} \sim_{E} e_{2}$, then

$$
\mathcal{H}\left(e_{1}\right) \sim_{C} \mathcal{H}\left(e_{2}\right), \quad \mathcal{T}\left(e_{1}\right) \sim_{C} \mathcal{T}\left(e_{2}\right) .
$$

Observe that self-coupling is permitted (that is, we allow $\mathcal{H}(e)=\mathcal{T}(e)$ ) and multiple arrows are permitted (it is possible to have $\mathcal{H}\left(e_{1}\right)=\mathcal{H}\left(e_{2}\right)$ and $\mathcal{T}\left(e_{1}\right)=\mathcal{T}\left(e_{2}\right)$ for $\left.e_{1} \neq e_{2}\right)$.

Associated with each cell $c \in \mathcal{C}$ is an important set of edges, namely, those that will be interpreted as representing couplings into cell $c$.

Definition 2.2. Let $c \in \mathcal{C}$. Then the input set of $c$ is

$$
I(c)=\{e \in \mathcal{E}: \mathcal{H}(e)=c\} .
$$

An element of $I(c)$ is called an input edge or input arrow of $c$.

The following concept is fundamental.

Definition 2.3. The relation $\sim_{I}$ of input equivalence on $\mathcal{C}$ is defined by $c \sim_{I} d$ if and only if there exists an arrow-type preserving bijection

$$
\beta: I(c) \rightarrow I(d)
$$

That is, for every input arrow $i \in I(c)$

$$
i \sim_{E} \beta(i) .
$$

Any such bijection $\beta$ is called an input isomorphism from cell $c$ to cell $d$. The set $B(c, d)$ denotes the collection of all input isomorphisms from cell $c$ to cell $d$. The set

$$
\mathcal{B}_{\mathcal{G}}=\bigcup_{c, d \in \mathcal{C}} B(c, d)
$$

is a groupoid (Brandt [1], Brown [2], Higgins [8]), which is an algebraic structure rather like a group, except that the product of two elements is not always defined. We call $\mathcal{B}_{\mathcal{G}}$ the groupoid of the network. Note that the union in (2.4) is disjoint and that $B(c, c)$ is a permutation group acting on the input set $I(c)$.

The definitions of input equivalence, input isomorphism, and groupoid of the network are direct generalizations to the multiarrow context of Definitions 3.2 and 3.5 in [10]. By the consistency condition (f) of Definition 2.1, $c \sim_{I} d$ implies $c \sim_{C} d$, but the converse fails in general.

Remark 2.4. (a) Suppose that a cell $c$ has two edge equivalent input arrows $i, j \in I(c)$, that is, $i \sim_{E} j$. Then the transposition $(i j)$ is an input isomorphism in $B(c, c)$. 
(b) The reason for introducing an explicit set $I(c)$ of input arrows is to provide a welldefined set for the input isomorphism $\beta$ in (2.2) to act on. Otherwise we must consider "sets" in which elements may occur more than once. This is the main novelty in Definition 2.3 compared to that in [10].

There does exist a standard theory of such "sets," which are called multisets. See Wildberger [12]. The multiarrow formalism could also be set up in multiset language.

Definition 2.5. A homogeneous network is a coupled cell network such that $B(c, d) \neq \emptyset$ for every pair of cells $c, d$.

3. Vector fields on a coupled cell network. We now define the class $\mathcal{F}_{G}^{P}$ of admissible vector fields corresponding to a given coupled cell network $\mathcal{G}$. This class consists of all vector fields that are "compatible" with the labeled graph structure or, equivalently, are "symmetric" under the groupoid $\mathcal{B}_{\mathcal{G}}$. It also depends on a choice of "total phase space" $P$, which we assume is fixed throughout the subsequent discussion.

For each cell in $\mathcal{C}$ define a cell phase space $P_{c}$. This must be a smooth manifold of dimension $\geq 1$, which for simplicity we assume is a nonzero finite-dimensional real vector space. We require

$$
c \sim_{C} d \quad \Longrightarrow \quad P_{c}=P_{d}
$$

and we employ the same coordinate systems on $P_{c}$ and $P_{d}$. Only these identifications of cell phase spaces are canonical; that is, the relation $c \sim_{C} d$ implies that cells $c$ and $d$ have the same phase space but not that they have isomorphic (conjugate) dynamics.

Define the corresponding total phase space to be

$$
P=\prod_{c \in \mathcal{C}} P_{c}
$$

and employ the coordinate system

$$
x=\left(x_{c}\right)_{c \in \mathcal{C}}
$$

on $P$.

More generally, suppose that $\mathcal{D}=\left(d_{1}, \ldots, d_{s}\right)$ is any finite ordered subset of $s$ cells in $\mathcal{C}$. In particular, the same cell can appear more than once in $\mathcal{D}$. Define

$$
P_{\mathcal{D}}=P_{d_{1}} \times \cdots \times P_{d_{s}}
$$

Further, write

$$
x_{\mathcal{D}}=\left(x_{d_{1}}, \ldots, x_{d_{s}}\right),
$$

where $x_{d_{j}} \in P_{d_{j}}$.

For a given cell $c$ the internal phase space is $P_{c}$ and the coupling phase space is

$$
P_{\mathcal{T}(I(c))}=P_{\mathcal{T}\left(i_{1}\right)} \times \cdots \times P_{\mathcal{T}\left(i_{s}\right)},
$$


where $\mathcal{T}(I(c))$ denotes the ordered set of cells $\left(\mathcal{T}\left(i_{1}\right), \ldots, \mathcal{T}\left(i_{s}\right)\right)$ as the arrows $i_{k}$ run through $I(c)$. Suppose $c, d \in \mathcal{C}$ and $c \sim_{I} d$. For any $\beta \in B(c, d)$, define the pullback map

$$
\beta^{*}: P_{\mathcal{T}(I(d))} \rightarrow P_{\mathcal{T}(I(c))}
$$

by

$$
\left(\beta^{*}(z)\right)_{\mathcal{T}(i)}=z_{\mathcal{T}(\beta(i))}
$$

for all $i \in I(c)$ and $z \in P_{\mathcal{T}(I(d))}$. We use pullback maps to relate different components of a vector field associated with a given coupled cell network. Specifically, the class of vector fields that is encoded by a coupled cell network is given in Definition 3.1.

Definition 3.1. A vector field $f: P \rightarrow P$ is $\mathcal{B}_{G}$-equivariant or $\mathcal{G}$-admissible if the following hold:

(a) For all $c \in \mathcal{C}$ the component $f_{c}(x)$ depends only on the internal phase space variables $x_{c}$ and the coupling phase space variables $x_{\mathcal{T}(I(c))}$; that is, there exists $\hat{f}_{c}: P_{c} \times P_{\mathcal{T}(I(c))} \rightarrow$ $P_{c}$ such that

$$
f_{c}(x)=\hat{f}_{c}\left(x_{c}, x_{\mathcal{T}(I(c))}\right) .
$$

(b) For all $c, d \in \mathcal{C}$ and $\beta \in B(c, d)$

$$
\hat{f}_{d}\left(x_{d}, x_{\mathcal{T}(I(d))}\right)=\hat{f}_{c}\left(x_{d}, \beta^{*}\left(x_{\mathcal{T}(I(d))}\right)\right)
$$

for all $x \in P$.

Observe that self-coupling is allowed (that is, $P_{c}$ can be one of the factors in $P_{\mathcal{T}(I(c))}$ ) and multiple arrows between two cells are allowed (since the tail of two arrows terminating in $I(c)$ can be the same cell). However, when repetition occurs, the repeated coordinates are always identical.

It follows that $f$ is determined if we specify one mapping for each input equivalence class of cells. Indeed, each admissible vector field on a homogeneous cell system is uniquely determined by a single mapping $f_{c}$ at some node $c$. In general, each component $f_{c}$ of $f$ is invariant under the vertex group $B(c, c)$. Indeed, every such invariant function determines a unique admissible vector field.

ODE-equivalent networks. In the enlarged class of coupled cell networks, it is possible for two different coupled cell systems $\mathcal{G}_{1}$ and $\mathcal{G}_{2}$ to generate the same space of admissible vector fields. For instance, consider the two two-cell systems in Figure 5. Their corresponding systems of admissible vector fields are

$$
\begin{aligned}
\dot{x_{1}} & =g\left(x_{1}, x_{1}, x_{2}\right), \\
\dot{x_{2}} & =g\left(x_{2}, x_{2}, x_{1}\right)
\end{aligned} \quad \text { and } \quad \begin{aligned}
\dot{x_{1}} & =f\left(x_{1}, x_{2}\right), \\
\dot{x_{2}} & =f\left(x_{2}, x_{1}\right) .
\end{aligned}
$$

These two-cell networks clearly define the same spaces of admissible vector fields. Indeed, given $f$ we can set $g(x, y, z)=f(x, z)$, and given $g$ we can set $f(a, b)=g(a, a, b)$. A testable condition for determining ODE-equivalence is given by Dias and Stewart [4] who show that two networks are ODE-equivalent if and only if the corresponding spaces of linear admissible vector fields are identical. 


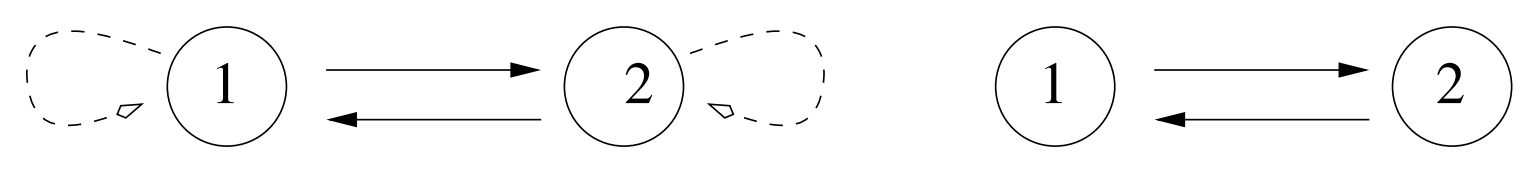

Figure 5. Two ODE-equivalent networks.

4. Balanced equivalence relations. We now extend the key concept of a balanced equivalence relation to the multiarrow formalism and generalize its properties.

Definition 4.1. An equivalence relation $\bowtie$ on $\mathcal{C}$ is balanced if for every $c, d \in \mathcal{C}$ with $c \bowtie d$, there exists an input isomorphism $\beta \in B(c, d)$ such that $\mathcal{T}(i) \bowtie \mathcal{T}(\beta(i))$ for all $i \in I(c)$.

In particular, $B(c, d) \neq \emptyset$ implies $c \sim_{I} d$. Hence, balanced equivalence relations refine $\sim_{I}$.

In the important special case where all pairs of arrows connecting the same two cells are $\sim_{E}$-equivalent, there is a graphical way to test whether a given equivalence relation $\bowtie$ is balanced. Color the cells in a network so that two cells have the same color precisely when they are in the same $\bowtie$-equivalence class. Then $\bowtie$ is balanced if and only if every pair of identically colored cells admits a color-preserving input isomorphism (more precisely, an input isomorphism $\beta$ where $\mathcal{T}(i)$ and $\mathcal{T}(\beta(i))$ have the same color). For example, consider the balanced relation in the network in Figure 1 (right).

Choose a total phase space $P$, and let $\bowtie$ be an equivalence relation on $\mathcal{C}$. We assume that $\bowtie$ is a refinement of $\sim_{\mathcal{C}}$; that is, if $c \bowtie d$, then $c$ and $d$ have the same cell labels. It follows that the polydiagonal subspace

$$
\Delta_{\bowtie}=\left\{x \in P: x_{c}=x_{d} \text { whenever } c \bowtie d \quad \forall c, d \in \mathcal{C}\right\}
$$

is well defined, since $x_{c}$ and $x_{d}$ lie in the same space $P_{c}=P_{d}$. The polydiagonal $\Delta_{\bowtie}$ is a linear subspace of $P$.

Definition 4.2. Let $\bowtie$ be an equivalence relation on $\mathcal{C}$. Then $\bowtie$ is robustly polysynchronous if $\Delta_{\bowtie}$ is invariant under every vector field $f \in \mathcal{F}_{G}^{P}$. That is,

$$
f\left(\Delta_{\bowtie}\right) \subseteq \Delta_{\bowtie}
$$

for all $f \in \mathcal{F}_{G}^{P}$. Equivalently, if $x(t)$ is a trajectory of any $f \in \mathcal{F}_{G}^{P}$, with initial condition $x(0) \in \Delta_{\bowtie}$, then $x(t) \in \Delta_{\bowtie}$ for all $t \in \mathbf{R}$.

We now generalize Theorem 6.5 of [10] to the multiarrow formalism.

Theorem 4.3. Let $\bowtie$ be an equivalence relation on a coupled cell network. Then $\bowtie$ is robustly polysynchronous if and only if $\bowtie$ is balanced.

Proof. The proof is essentially the same as that of Theorem 6.5 of [10]. The main points are that it is easy to check directly that $\bowtie$ being balanced is sufficient for $\Delta_{\bowtie}$ to be robustly polysynchronous, while necessity can be established by considering admissible linear vector fields. We take these points in turn.

First, suppose that $\bowtie$ is balanced, and let $f \in \mathcal{F}_{G}^{P}$. Suppose that $c \bowtie d$. By Definition 4.1 the set $B(c, d)$ is nonempty, so there exists $\beta \in B(c, d)$. We have $\beta(c)=d$.

We know that for all $c \in \mathcal{C}$ the component $f_{c}(x)$ is symmetric under all permutations of the input set $I(c)$ that preserve $\bowtie$-equivalence classes. Therefore, for any $x \in \Delta_{\bowtie}$,

$$
f_{d}(x)=\hat{f}_{d}\left(x_{d}, x_{\mathcal{T}(I(d))}\right)=\hat{f}_{c}\left(x_{d}, \beta^{*}\left(x_{\mathcal{T}(I(d))}\right)\right)=\hat{f}_{c}\left(x_{c}, x_{\mathcal{T}(I(c))}\right)=f_{c}(x)
$$


because $\beta$ preserves the $\bowtie$-equivalence classes. Therefore, $f$ leaves $\Delta_{\bowtie}$ invariant.

For the converse, suppose that $\Delta_{\bowtie}$ is invariant under all $f \in \mathcal{F}_{G}^{P}$. Then, in particular, $\Delta_{\bowtie}$ is invariant under all linear $f \in \mathcal{F}_{G}^{P}$. Let $c \neq d \in \mathcal{C}$ with $c \bowtie d$. We first show that $c \sim_{I} d$. If not, we can define an admissible linear vector field $f$ such that $f_{c}=0, f_{d} \neq 0$. This contradicts invariance of $\Delta_{\bowtie}$. Therefore, $c \bowtie d$ implies that $c, d$ are input-equivalent as claimed.

Next, we construct a class of admissible linear vector fields as follows. For each pair of $\sim_{C}$-equivalence classes of cells $([c],[d])$ choose representatives $c, d \in \mathcal{C}$. Choose some linear map

$$
\lambda_{d c}: P_{d} \rightarrow P_{c}
$$

If $c^{\prime} \sim_{C} c$ and $d^{\prime} \sim_{C} d$, use the canonical identifications of $P_{c^{\prime}}$ with $P_{c}$ and $P_{d^{\prime}}$ with $P_{d}$ to pull back $\lambda_{d c}$ to a linear map

$$
\lambda_{d^{\prime} c^{\prime}}: P_{d^{\prime}} \rightarrow P_{c^{\prime}}
$$

That is, we ensure that $\lambda_{d c}$ remains "the same" map when cells are replaced by canonically identified cells.

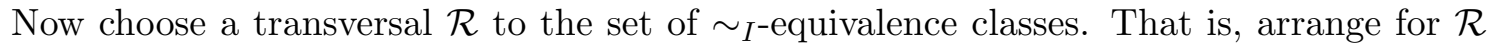
to contain precisely one member of each $\sim_{I^{-}}$equivalence class. For each $t \in \mathcal{R}$ define

$$
\Lambda_{t}(x)=\sum_{i \in I(t)} \lambda_{\mathcal{T}(i) t}\left(x_{\mathcal{T}(i)}\right)
$$

If $i, j \in I(t)$ and $i \sim_{E} j$, impose the extra condition

$$
\lambda_{\mathcal{T}(i) t}=\lambda_{\mathcal{T}(j) t}
$$

where we canonically identify $P_{\mathcal{T}(i)}$ with $P_{\mathcal{T}(j)}$. Condition (4.1) ensures that $\Lambda_{t}$ is $B(t, t)$ invariant.

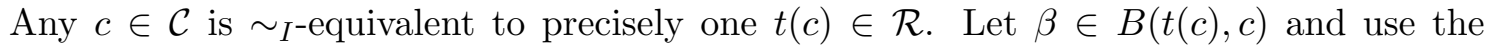
pullback $\beta^{*}$ to define

$$
\Lambda_{c}(x)=\Lambda_{\beta(t(c))}(x)=\Lambda_{t(c)}\left(\beta^{*}(x)\right) .
$$

The $B(t, t)$-invariance of $\Lambda_{t(c)}$ implies that all $\beta \in B(t(c), c)$ lead to the same $\Lambda_{c}$. Lemma 4.5 of [10], trivially extended to the multiarrow formalism, implies that $\Lambda$ is $\mathcal{B}_{G}$-equivariant, that is, admissible.

The final preparatory step is to partition the input sets $I(c)$ according to the $\sim_{E}$-equivalence classes of arrows. Full details (which easily generalize to the multiarrow formalism) are at the end of section 3 of [10] under the heading "Structure of $B(c, d)$." Introduce an equivalence relation $\equiv_{c}$ on $I(c)$ for which

$$
j_{1} \equiv_{c} j_{2} \quad \Longleftrightarrow \quad j_{1} \sim_{E} j_{2}
$$

That is, $\equiv_{c}$ is the restriction of $\sim_{E}$ to $I(c)$. Let the $\equiv_{c^{-}}$equivalence classes be $K_{0}^{c}, \ldots, K_{r}^{c}$ for $r=r(c)$. By convention $K_{0}^{c}=\{c\}$. By section 3 of [10] the vertex group $B(c, c)$ is isomorphic to the direct product of symmetric groups $\mathbf{S}_{k_{j}^{c}}$ acting on the sets $K_{j}^{c}$, where $k_{j}^{c}=\left|K_{j}^{c}\right|$. 
Let the $\bowtie$-equivalence classes be $A_{1}, \ldots, A_{m}$. Let $X_{l}$ denote the common value of the components $x_{i}$ for $i \in A_{l}$. Let $\mu_{s}^{c}$ denote the common value of the $\lambda_{\mathcal{T}(j) t(c)}$ for $j \in K_{s}^{c}$.

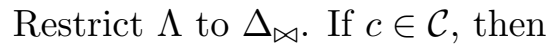

$$
\begin{aligned}
\Lambda_{c}(x) & =\sum_{j \in I(c)} \lambda_{\mathcal{T}(j) t(c)}\left(x_{\mathcal{T}(j)}\right) \\
& =\sum_{s=0}^{r(c)} \sum_{j \in K_{s}^{c}} \lambda_{\mathcal{T}(j) t(c)}\left(x_{\mathcal{T}(j)}\right) \\
& =\sum_{s=0}^{r(c)} \sum_{l=1}^{m} \sum_{j \in K_{s}^{c} \cap \mathcal{T}^{-1}\left(A_{l}\right)} \lambda_{\mathcal{T}(j) t(c)}\left(x_{\mathcal{T}(j)}\right) \\
& =\sum_{s=0}^{r(c)} \sum_{l=1}^{m} \sum_{j \in K_{s}^{c} \cap \mathcal{T}^{-1}\left(A_{l}\right)} \mu_{s}^{c}\left(X_{l}\right) \\
& =\sum_{s=0}^{r(c)} \sum_{l=1}^{m}\left|K_{s}^{c} \cap \mathcal{T}^{-1}\left(A_{l}\right)\right| \mu_{s}^{c}\left(X_{l}\right) .
\end{aligned}
$$

Now suppose that $c \bowtie d$ with $c \neq d$. Since $\bowtie$ is robustly synchronous, $\Lambda_{c}$ and $\Lambda_{d}$ must

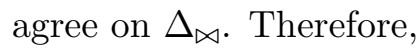

$$
\left|K_{s}^{c} \cap \mathcal{T}^{-1}\left(A_{l}\right)\right|=\left|K_{s}^{d} \cap \mathcal{T}^{-1}\left(A_{l}\right)\right|
$$

whenever $0 \leq s \leq r(c)=r(d)$ and $1 \leq l \leq m$. This is the "cardinality condition" (6.2) of [10], and it clearly implies that $\bowtie$ is balanced (use the fact that $B(c, c) \cong \mathbf{S}_{k_{1}^{c}} \times \cdots \times \mathbf{S}_{k_{r(c)}^{c}}$, as in the proof of Theorem 6.5 of [10]).

5. Quotient networks. In this section we show that each balanced equivalence relation $\bowtie$ of a coupled cell network $\mathcal{G}$ induces a unique canonical coupled cell network $\mathcal{G}_{\bowtie}$ on $\Delta_{\bowtie}$, called the quotient network. This was not the case in the setting of [10], where quotient networks always existed but where there was not always a unique canonical choice. It was shown in [10] in the context of coupled cell systems without self-coupling and multiple arrows that every admissible vector field on the original network restricts to an admissible vector field on $\Delta_{\bowtie}$ in every quotient network. However, in general admissible vector fields on a quotient network could not be extended to an admissible vector field on the original network.

In the present context admissible vector fields restrict to admissible vector fields and every admissible vector field on the canonical quotient $\mathcal{G}_{\bowtie}$ lifts to an admissible vector field on $\mathcal{G}$. We begin by defining the (canonical) quotient network.

To define a network (see Definition 2.1) we need to (A) specify the cells; (B) specify an equivalence relation on cells; (C) specify the arrows; (D) specify an equivalence relation on arrows; (E) define the heads and tails of arrows; and (F) prove a consistency relation between arrows and cells. We do each of these in turn.

(A) Let $\bar{c}$ denote the $\bowtie$-equivalence class of $c \in \mathcal{C}$. The cells in $\mathcal{C}_{\triangleright}$ are the $\bowtie$-equivalence classes in $\mathcal{C}$; that is,

$$
\mathcal{C}_{\bowtie}=\{\bar{c}: c \in \mathcal{C}\} .
$$


Thus we obtain $\mathcal{C}_{\bowtie}$ by forming the quotient of $\mathcal{C}$ by $\bowtie$, that is, $\mathcal{C}_{\bowtie}=\mathcal{C} / \bowtie$.

(B) Define

$$
\bar{c} \sim_{C_{\triangleright}} \bar{d} \Longleftrightarrow c \sim_{C} d .
$$

The relation $\sim_{C_{\triangleright}}$ is well defined since $\bowtie$ refines $\sim_{C}$.

(C) Let $\mathcal{S} \subset \mathcal{C}$ be a set of cells consisting of precisely one cell $c$ from each $\bowtie$-equivalence class. The input arrows for a quotient cell $\bar{c}$ are identified with the input arrows in cell $c$, where $c \in \mathcal{S}$, that is, $I(\bar{c})=I(c)$. When viewing the arrow $i \in I(c)$ as an arrow in $I(\bar{c})$, we denote that arrow by $\bar{i}$. Thus, the arrows in the quotient network are the projection of arrows in the original network formed by the disjoint union

$$
\mathcal{E}_{\bowtie}=\bigcup_{c \in \mathcal{S}} I(c) .
$$

We show below that the definition of the quotient network structure is independent of the choice of the representative cells $c \in \mathcal{S}$.

(D) Two quotient arrows are equivalent when the original arrows are equivalent. That is,

$$
\overline{i_{1}} \sim_{E_{\triangleright}} \overline{i_{2}} \quad \Longleftrightarrow \quad i_{1} \sim_{E} i_{2}
$$

where $i_{1} \in I\left(c_{1}\right), i_{2} \in I\left(c_{2}\right)$, and $c_{1}, c_{2} \in \mathcal{S}$.

(E) Define the heads and tails of quotient arrows by

$$
\mathcal{H}(\bar{i})=\overline{\mathcal{H}(i)}, \quad \mathcal{T}(\bar{i})=\overline{\mathcal{T}(i)}
$$

(F) We now verify that the quotient network satisfies the consistency condition Definition 2.1(f). Note that (5.2) implies that when two arrows $\overline{i_{1}}$ and $\overline{i_{2}}$ in $\mathcal{E}_{\bowtie}$ are $\sim_{E_{\bowtie}}$ equivalent, their head and tail cells satisfy $\mathcal{H}\left(i_{1}\right) \sim_{C} \mathcal{H}\left(i_{2}\right)$ and $\mathcal{T}\left(i_{1}\right) \sim_{C} \mathcal{T}\left(i_{2}\right)$. Therefore, $\overline{\mathcal{H}\left(i_{1}\right)} \sim_{C_{\bowtie}} \overline{\mathcal{H}\left(i_{2}\right)}$ and $\overline{\mathcal{T}\left(i_{1}\right)} \sim_{C_{\bowtie}} \overline{\mathcal{T}\left(i_{2}\right)}$. This implies $\mathcal{H}\left(\overline{i_{1}}\right) \sim_{C_{\triangleright}} \mathcal{H}\left(\overline{i_{2}}\right)$ and $\mathcal{T}\left(\overline{i_{1}}\right) \sim_{C_{\bowtie}} \mathcal{T}\left(\overline{i_{2}}\right)$, as desired.

Independence of quotient network on choice of cells in $\mathcal{S}$. We claim that, because $\bowtie$ is balanced, choosing different representatives in $\mathcal{S}$ of the $\bowtie$-equivalence classes leads to isomorphic quotient networks. Indeed, suppose $c_{1} \bowtie c_{2}$. By Definition 4.1, there is an (arrow-type preserving) input isomorphism $\beta: I\left(c_{1}\right) \rightarrow I\left(c_{2}\right)$ that preserves the $\bowtie$-class of the tails. This induces a bijection between $I\left(\overline{c_{1}}\right)=\left\{\bar{i}: i \in I\left(c_{1}\right)\right\}$ and $I\left(\overline{c_{2}}\right)=\left\{\bar{j}: j \in I\left(c_{2}\right)\right\}$ (and, therefore, between the arrow sets of the two quotient networks constructed using $c_{1}$ or $c_{2}$ as a representative of this $\bowtie$-equivalence class) that identifies the two networks in a consistent manner: $\bar{i}$ and $\overline{\beta(i)}$ are in the same arrow-equivalence class, $\mathcal{H}(\bar{i})=\mathcal{H}(\overline{\beta(i)})$, and $\mathcal{T}(\bar{i})=\mathcal{T}(\overline{\beta(i)})$.

Remark 5.1. (a) Note that when $c_{1} \bowtie c_{2}$, any input arrow in $I\left(c_{2}\right)$ with tail cell $c_{1}$ leads to a self-coupling arrow in the quotient. If $c_{1}$ and $c_{2}$ are distinct $\bowtie$-equivalent cells having equivalent arrows with the same head cell $d$, then multiarrows will be present in the quotient network, where a multiarrow is a set of several edge-equivalent arrows between two given cells. For example, see Figure 1 (right) and the corresponding quotient network Figure 2 (right).

(b) Input isomorphisms on $\mathcal{G}$ project onto input isomorphisms of $\mathcal{G}_{\bowtie}$. Let $\beta: I(c) \rightarrow I(d)$ be an input isomorphism between input sets of cells $c$ and $d$. Then $\beta: I(\bar{c}) \rightarrow I(\bar{d})$ is also 
a bijection since $I(c)=I(\bar{c})$ and $I(d)=I(\bar{d})$. Identity (5.2) guarantees that (2.3) is valid

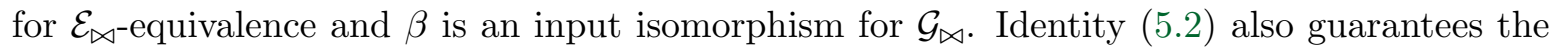
converse - every input equivalence on $\mathcal{G}_{\bowtie}$ lifts to one on $\mathcal{G}$.

(c) Since input isomorphisms project, we see that any quotient of a homogeneous network is also a homogeneous cell network. The quotient of the balanced relation of the five-cell example in Figures 1 and 2 (left) shows that this remark is not valid for quotients in the class of networks considered in [10].

We can now generalize Theorem 9.2 of [10] to the multiarrow formalism. The fact that every vector field on the quotient lifts to a vector field on the original network is a major theoretical reason for introducing this new formalism.

Theorem 5.2. Let $\bowtie$ be a balanced relation on a coupled cell network $\mathcal{G}$.

(a) The restriction of a $\mathcal{G}$-admissible vector field to $\Delta_{\bowtie}$ is $\mathcal{G}_{\bowtie}$-admissible.

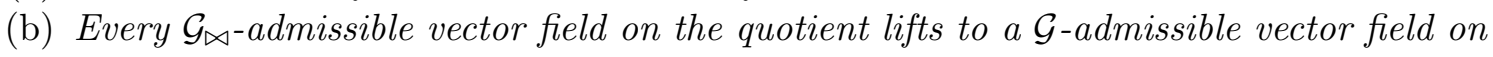
the original network.

Proof. (a) The proof of Theorem 5.2(a) is identical to the proof of Theorem 9.2 of [10].

(b) Let $\bar{c}$ be a quotient cell and suppose that the dynamics on that cell are prescribed by the ODE

$$
\dot{x}_{\bar{c}}=\hat{f}_{\bar{c}}\left(x_{\bar{c}}, x_{\mathcal{T}(I(\bar{c}))}\right),
$$

where $x_{\bar{c}} \in P_{\bar{c}}=P_{c}$ are the internal state space variables and $x_{\mathcal{T}(I(\bar{c}))} \in P_{\mathcal{T}(I(\bar{c}))}=P_{\mathcal{T}(I(c))}$ are the coupling variables. We can lift this ODE to each cell $c$ that quotients onto $\bar{c}$ by

$$
\dot{x}_{c}=\hat{f}_{\bar{c}}\left(x_{c}, x_{\mathcal{T}(I(c))}\right) .
$$

Observe that if $c \bowtie d($ or $\bar{c}=\bar{d})$, then there exists an input isomorphism $\beta: I(c) \rightarrow I(d)$. Now

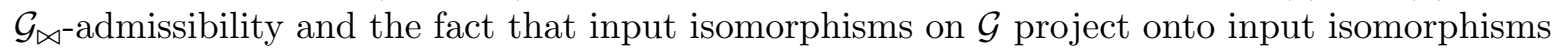
on $\mathcal{G}_{\bowtie}$ imply that

$$
\hat{f}_{\bar{d}}\left(x_{d}, x_{\mathcal{T}(I(\bar{d}))}\right)=\hat{f}_{\bar{c}}\left(x_{d}, \beta^{*}\left(x_{\mathcal{T}(I(\bar{c}))}\right)\right) .
$$

Note that if $c=d$, then (5.3) is consistent since $f_{\bar{c}}$ is invariant under $B(\bar{c}, \bar{c})$. Therefore,

$$
\hat{f}_{d}\left(x_{d}, x_{\mathcal{T}(I(d))}\right)=\hat{f}_{c}\left(x_{d}, \beta^{*}\left(x_{\mathcal{T}(I(d))}\right)\right),
$$

and the lift (5.3) is $\mathcal{G}$-admissible.

6. Examples of networks. Several examples of networks with interesting properties were presented in [7]. The simplest network with self-coupling is the feed-forward network shown in Figure 6. This network has a surprising bifurcation structure. It is shown in [7] that synchronybreaking bifurcations occur with multiple eigenvalues (and nontrivial Jordan normal form) in codimension one. Suppose that $\lambda$ is the bifurcation parameter and that Hopf bifurcation occurs at $\lambda=0$. Then this bifurcation leads to periodic solutions whose amplitude growth is the expected $\lambda^{1 / 2}$ in cell 2 , but is a surprising $\lambda^{1 / 6}$ in cell 3 .

Leite and Golubitsky [9] classify all homogeneous three-cell networks with two input arrows at each node. See Figure 8. There are 34 different networks. We have seen previously that 


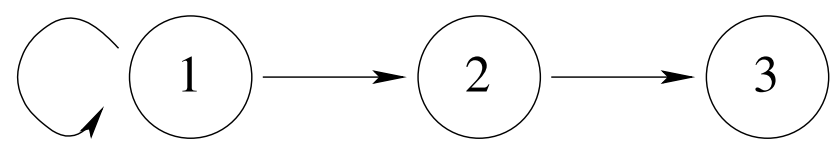

Figure 6. The three-cell feed-forward network.

quotient networks can have multiple arrows even when the original network does not. For example the five-cell network in Figure 1 has network 29 in Figure 8 as a quotient network. Another example (that was discussed in [10]) is the balanced coloring in the five-cell network in Figure 7 whose quotient is the three-cell bidirectional ring (34 in Figure 8) with $\mathbf{D}_{3}$ symmetry. In section 8 we prove that every (identical-edge) homogeneous network is a quotient of a homogeneous network without self-coupling and multiple arrows.

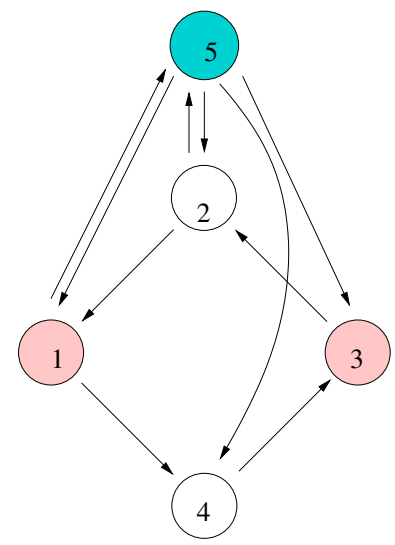

Figure 7. A second homogeneous five-cell network with balanced coloring.

It is also shown in [9] that steady-state, codimension-one, synchrony-breaking bifurcations of the networks in Figure 8 can occur with simple real eigenvalues, real double eigenvalues with independent eigenvectors (as in the bidirectional ring), real double eigenvalues with nontrivial Jordan blocks (as in the feed-forward network in Figure 6), or with complex-conjugate, purely imaginary eigenvalues (as in the three-cell unidirectional ring).

Planar lattice dynamical systems with nearest neighbor coupling have interesting patterns of synchrony. For example, [7] shows that there exists an infinite family of balanced two colorings, almost all of which are not spatially periodic. Wang and Golubitsky [11] classify all balanced two-color patterns with nearest neighbor coupling (NN) and with both nearest and next nearest neighbor coupling (NNN). The classification proceeds by assuming the form of the two-cell quotient and then classifying all balanced colorings that lead to that quotient. In NNN, all balanced relations are spatially doubly periodic (which is strikingly different from the NN), thus illustrating again the importance of network architecture.

7. Hyperbolic equilibria and balanced relations. Theorem 4.3 shows that balanced equivalence relations determine the robust patterns of synchrony on a given network - that is, those patterns that are determined by flow-invariant subspaces (for all admissible vector fields). A fundamental question concerns how patterns of synchrony for a given network can be estab- 
1.
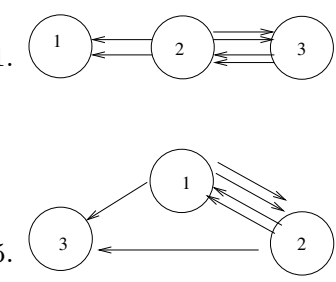

9.

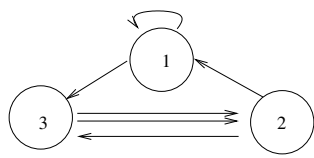

13. $(1) \rightleftarrows 3$

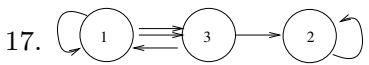

21.
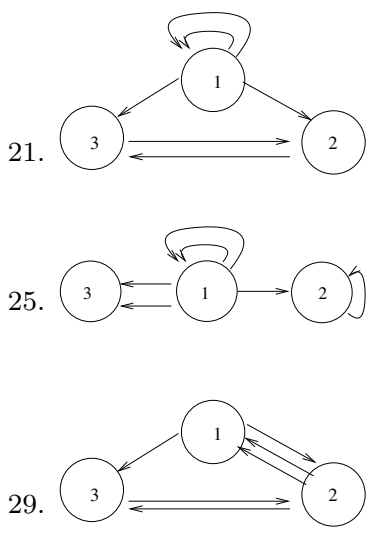

30.

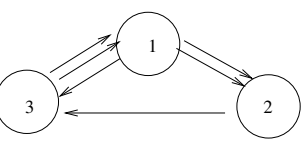

10.
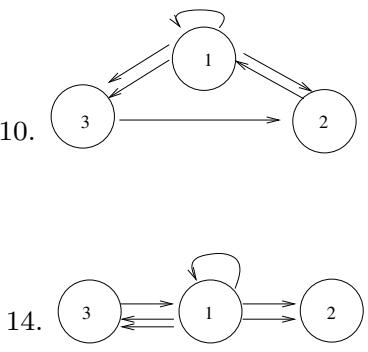

15.

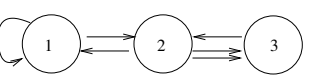

7. 1132

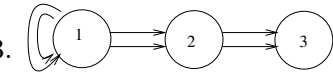

4.

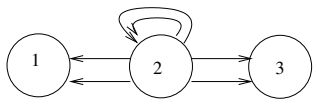

11.

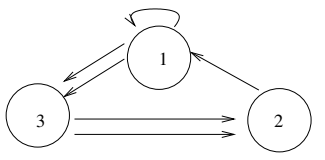

12. $( 1 ) = 2 \longdiv { 2 }$

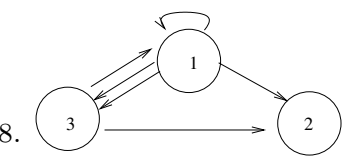

16.

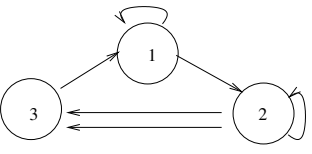

20.

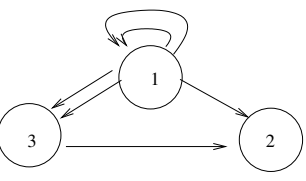

23.

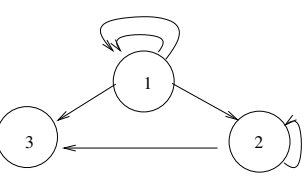

24.

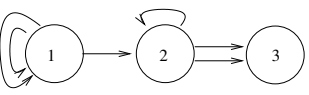

27. $(19) \rightarrow 3=2$

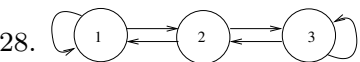

31.

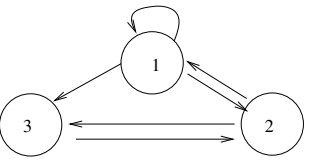

32 .

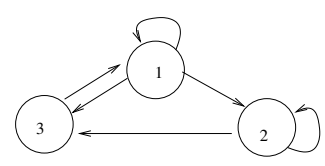

33.

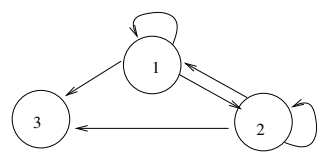

Figure 8. Homogeneous three-cell networks with two input arrows at each node from [9].

lished in practice. One presumption is that such patterns will be observed through synchronized dynamical states in simulation (or experiment). Moreover, in an abstract perfect world, it is also reasonable to presume that patterns of synchrony will be observed only if the patterns are unchanged by small perturbation (either in initial conditions or in changes in parameters). We call a pattern "rigid" if it does not change when the vector field is perturbed by all suf- 
ficiently small admissible perturbations. In this section we prove that patterns of synchrony associated to hyperbolic equilibria are rigid precisely when they are balanced. Thus, in coupled cell networks, the local assumption of rigidity for patterns associated to one equilibrium for each of a small but open set of vector fields implies the global invariance of a polydiagonal subspace for all admissible vector fields. We conjecture that a similar statement is valid for hyperbolic periodic states, but we are currently unable to prove this conjecture.

Let $x_{0}=\left(x_{1}^{0}, \ldots, x_{N}^{0}\right) \in P$. Define the equivalence relation $\equiv_{x_{0}}$ by $c \equiv_{x_{0}} d$ if and only if $c \sim_{C} d$ and $x_{c}^{0}=x_{d}^{0}$. (This notation does not conflict with our previous use of $\equiv_{c}$ in the proof of Theorem 4.3, which was temporary notation for that proof.) Suppose that we color two cells $c$ and $d$ the same color if and only if $c \equiv_{x_{0}} d$. Then this coloring is the pattern of synchrony associated to $x_{0}$. Note that

$$
\Delta_{\equiv_{x_{0}}}=\left\{x \in P: x_{c}=x_{d} \text { if } \quad c \equiv_{x_{0}} d\right\}
$$

is the smallest subspace of $P$ that contains all points with the same pattern of synchrony.

Definition 7.1. Let $x_{0} \in P$ be a hyperbolic equilibrium of a $C^{1}$-admissible cell system. The equivalence relation $\equiv_{x_{0}}$ is rigid if in each $C^{1}$ perturbed admissible system the hyperbolic equilibrium near $x_{0}$ remains in $\Delta_{\equiv_{x_{0}}}$. We also say that the pattern of synchrony defined by $x_{0}$ is rigid.

Strong admissibility. In Theorem 7.6 we prove that only those patterns of synchrony that are generated by balanced relations are rigid. We prove this theorem by showing that rigid patterns of synchrony lead to flow-invariant subspaces. The following is a key idea in the proof.

Definition 7.2. A map $G: P \rightarrow P$ is strongly admissible if $G_{c}(x)=G_{c}\left(x_{c}\right)$ for every cell $c$ and $G_{c}=G_{d}$ for every pair of cells where $c \sim_{C} d$.

A strongly admissible map $G$ is admissible since $c \sim_{I} d$ implies $c \sim_{C} d$ and hence $G_{c}=G_{d}$.

Lemma 7.3. Let $F: P \rightarrow P$ be admissible and let $G: P \rightarrow P$ be strongly admissible. Then $F \circ G$ and $G \circ F$ are admissible.

Proof. Both $(F \circ G)_{c}$ and $(G \circ F)_{c}$ are functions defined on $P_{c} \times P_{\mathcal{T}(I(c))}$. That is,

$$
\begin{aligned}
(G \circ F)_{c}\left(x_{c}, x_{\mathcal{T}(I(c))}\right) & =G_{c}\left(F_{c}\left(x_{c}, x_{\mathcal{T}(I(c))}\right)\right) \\
(F \circ G)_{c}\left(x_{c}, x_{\mathcal{T}(I(c))}\right) & =F_{c}\left(G_{c}\left(x_{c}\right), G_{\mathcal{T}\left(i_{1}\right)}\left(x_{\mathcal{T}\left(i_{1}\right)}\right), \ldots, G_{\mathcal{T}\left(i_{s}\right)}\left(x_{\mathcal{T}\left(i_{s}\right)}\right)\right)
\end{aligned}
$$

where $I(c)=\left\{i_{1}, \ldots, i_{s}\right\}$.

Let $\beta: I(c) \rightarrow I(d)$ be an input isomorphism in $B(c, d)$. Order $I(d)=\left\{j_{1}, \ldots, j_{s}\right\}$ so that $\beta\left(i_{k}\right)=j_{k}$. It follows from the definition of input isomorphism that $c \sim_{I} d$ and $\mathcal{T}\left(i_{k}\right) \sim_{C} \mathcal{T}\left(j_{k}\right)$ for each $k$. Hence, $F_{c}=F_{d}, G_{c}=G_{d}$, and $G_{\mathcal{T}\left(i_{k}\right)}=G_{\mathcal{T}\left(j_{k}\right)}$.

We claim that both $(F \circ G)_{c}$ and $(G \circ F)_{c}$ are $\beta$ related to $(F \circ G)_{d}$ and $(G \circ F)_{d}$. To verify this point for $G \circ F$, compute

$$
\begin{aligned}
(G \circ F)_{d}\left(x_{d}, x_{\mathcal{T}(I(d))}\right) & =G_{d}\left(F_{d}\left(x_{d}, x_{\mathcal{T}(I(d))}\right)\right) \\
& =G_{d}\left(F_{c}\left(x_{d}, \beta^{*} x_{\mathcal{T}(I(d))}\right)\right) \\
& =G_{c}\left(F_{c}\left(x_{d}, \beta^{*} x_{\mathcal{T}(I(d))}\right)\right) \\
& =(G \circ F)_{c}\left(x_{d}, \beta^{*} x_{\mathcal{T}(I(d))}\right)
\end{aligned}
$$


Thus $G \circ F$ is admissible. It also follows that

$$
\begin{aligned}
(F \circ G)_{d}\left(x_{d}, x_{\mathcal{T}\left(j_{1}\right)}, \ldots, x_{\mathcal{T}\left(j_{s}\right)}\right) & =F_{d}\left(G_{d}\left(x_{d}\right), G_{\mathcal{T}\left(j_{1}\right)}\left(x_{\mathcal{T}\left(j_{1}\right)}\right), \ldots, G_{\mathcal{T}\left(j_{s}\right)}\left(x_{\mathcal{T}\left(j_{s}\right)}\right)\right) \\
& =F_{c}\left(G_{d}\left(x_{d}\right), G_{\mathcal{T}\left(j_{1}\right)}\left(x_{\mathcal{T}\left(j_{1}\right)}\right), \ldots, G_{\mathcal{T}\left(j_{s}\right)}\left(x_{\mathcal{T}\left(j_{s}\right)}\right)\right) \\
& =F_{c}\left(G_{c}\left(x_{d}\right), G_{\mathcal{T}\left(i_{1}\right)}\left(x_{\mathcal{T}\left(j_{1}\right)}\right), \ldots, G_{\mathcal{T}\left(i_{s}\right)}\left(x_{\mathcal{T}\left(j_{s}\right)}\right)\right) \\
& =(F \circ G)_{c}\left(x_{d}, x_{\mathcal{T}\left(j_{1}\right)}, \ldots, x_{\mathcal{T}\left(j_{s}\right)}\right) .
\end{aligned}
$$

Thus $F \circ G$ is also admissible.

Definition 7.4. Let $\bowtie$ be an equivalence relation. A point $x=\left(x_{1}, \ldots, x_{N}\right) \in \Delta_{\bowtie}$ is generic if $x_{i}=x_{j}$ for $i \sim_{C} j$ implies that $i \sim_{\bowtie j} j$.

Observe that generic points are open and dense in $\Delta_{\bowtie \text {. }}$

Lemma 7.5. Let $\bowtie$ be an equivalence relation on cells. Let $x_{0}$ be a generic point in $\Delta_{\bowtie}$ and let $y_{0}$ be any point in $\Delta_{\bowtie}$. Then there exists a strongly admissible map $G: P \rightarrow P$ such that $G\left(x_{0}\right)=y_{0}$.

Proof. Let $x_{0}=\left(x_{1}, \ldots, x_{N}\right)$ and $y_{0}=\left(y_{1}, \ldots, y_{N}\right)$. We need to choose functions $G_{c}$ (where $G_{c}=G_{d}$ whenever $c \sim_{C} d$ ) so that

$$
G_{c}\left(x_{c}\right)=y_{c}
$$

Conditions (7.1) are incompatible only when $c \sim_{C} d, x_{c}=x_{d}$, and $y_{c} \neq y_{d}$. When these conditions are compatible we can always choose strongly admissible interpolation functions $G_{c}$ to satisfy (7.1). The facts that $x_{0}, y_{0} \in \Delta_{\bowtie}$ and $x_{0}$ is generic ensure that conditions (7.1) are compatible because $x_{c}=x_{d}$ implies $y_{c}=y_{d}$.

Perturbation spaces and hyperbolic equilibria. Let $x_{0} \in P$. Form the subspace

$$
W_{x_{0}}=\left\{p\left(x_{0}\right): p \text { is admissible }\right\}
$$

consisting of all points obtained from $x_{0}$ by applying an admissible map. Let $\Delta \subset P$ be the smallest flow-invariant subspace that contains the point $x_{0}$. Flow-invariance implies that $\Delta=\Delta_{\bowtie_{x_{0}}}$ for some balanced equivalence relation $\bowtie_{x_{0}}$. The balanced equivalence relation

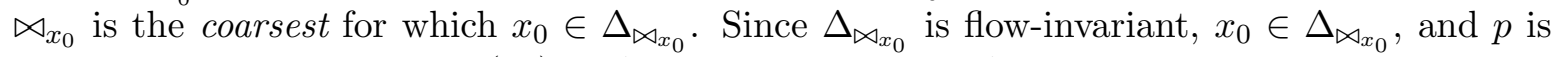
admissible, it follows that $p\left(x_{0}\right) \in \Delta_{\bowtie_{x_{0}}}$. Thus, $W_{x_{0}} \subset \Delta_{\bowtie_{x_{0}}}$. Equality need not hold, in general. However, equality does hold when the pattern of synchrony defined by a hyperbolic equilibrium $x_{0}$ is rigid.

Theorem 7.6. The equivalence relation $\equiv_{x_{0}}$ determined by the hyperbolic equilibrium $x_{0}$ is rigid if and only if $\equiv_{x_{0}}$ is balanced. Moreover, in this case, $\equiv_{x_{0}}=\bowtie_{x_{0}}$ and

$$
W_{x_{0}}=\Delta_{\equiv_{x_{0}}}=\Delta_{\bowtie_{x_{0}}}
$$

Proof. Let $x_{0} \in P$ be a hyperbolic equilibrium for a $C^{1}$-admissible vector field $f$ and assume that $\equiv_{x_{0}}$ is a balanced equivalence relation. It is straightforward to show that $\equiv_{x_{0}}$ is rigid. Hyperbolicity implies that every small admissible $C^{1}$ perturbation $g$ of $f$ will have a unique hyperbolic equilibrium $y_{0}$ near $x_{0}$. Since $\Delta_{\bowtie_{x_{0}}}$ is flow-invariant, uniqueness implies that $y_{0} \in \Delta_{\bowtie_{x_{0}}}$. Just restrict $f$ and $g$ to $\Delta_{\bowtie_{x_{0}}}$ and use hyperbolicity on this subspace. So the pattern of synchrony of the equilibrium $x_{0}$ is rigid. 
To prove the converse, we assume that $\equiv_{x_{0}}$ is a rigid equivalence relation. By the definition of $\equiv_{x_{0}}, x_{0}$ is generic in $\Delta_{\equiv_{x_{0}}}$. It follows from Lemma 7.5 that

$$
\Delta_{\equiv_{x_{0}}} \subset W_{x_{0}} .
$$

We claim that $\Delta_{\equiv_{x_{0}}}=W_{x_{0}}$. To verify this claim, let $p$ be an admissible vector field. Consider the perturbation $f_{\varepsilon}=f+\varepsilon p$ and denote by $x_{\varepsilon}$ the perturbed hyperbolic equilibrium for $f_{\varepsilon}$. So

$$
f_{\varepsilon}\left(x_{\varepsilon}\right)=0
$$

Since rigidity implies $x_{\varepsilon} \in \Delta_{\equiv_{x_{0}}}$, it follows that

$$
\left.\frac{d}{d \varepsilon} x_{\varepsilon}\right|_{\varepsilon=0} \in \Delta_{\equiv_{x_{0}}}
$$

Differentiating (7.4) with respect to $\varepsilon$ and evaluating at $\varepsilon=0$ yield

$$
0=\left.\frac{d}{d \varepsilon}\left(f\left(x_{\varepsilon}\right)+\varepsilon p\left(x_{\varepsilon}\right)\right)\right|_{\varepsilon=0}=\left.(D f)_{x_{0}} \frac{d}{d \varepsilon} x_{\varepsilon}\right|_{\varepsilon=0}+p\left(x_{0}\right) .
$$

Thus $p\left(x_{0}\right) \in(D f)_{x_{0}}\left(\Delta_{\equiv_{x_{0}}}\right)$; that is, $W_{x_{0}} \subset(D f)_{x_{0}}\left(\Delta_{\equiv_{x_{0}}}\right)$. In view of (7.3), we obtain

$$
\Delta_{\equiv_{x_{0}}} \subset W_{x_{0}} \subset(D f)_{x_{0}}\left(\Delta_{\equiv_{x_{0}}}\right) .
$$

Since the vector space $\Delta_{\equiv_{x_{0}}}$ is finite-dimensional, (7.5) implies that the inclusions above are all equalities, particularly $W_{x_{0}}=\Delta_{\equiv_{x_{0}}}$.

Next we show that $\Delta_{\equiv_{x_{0}}}$ is flow-invariant for all admissible vector fields. It then follows from Theorem 4.3 that $\equiv_{x_{0}}$ is a balanced relation, as desired. Let $y \in \Delta_{\equiv_{x_{0}}}$ and $q$ be an admissible vector field. We must show that $q(y) \in \Delta_{\equiv_{x_{0}}}$. By Lemma 7.5, $y=G\left(x_{0}\right)$ for some strongly admissible vector field $G$, and therefore $q(y)=q \circ G\left(x_{0}\right)$. By Lemma 7.3, $q \circ G$ is an admissible field, and therefore $q(y) \in W_{x_{0}}=\Delta_{\equiv_{x_{0}}}$.

Finally, we verify the moreover part of the theorem. Since $\Delta_{\equiv_{x_{0}}}$ is flow-invariant, it follows that $\Delta_{\bowtie_{x_{0}}} \subset \Delta_{\equiv_{x_{0}}}$. Since $W_{x_{0}} \subset \Delta_{\bowtie_{x_{0}}},(7.2)$ follows. Hence $\equiv_{x_{0}}=\bowtie_{x_{0}}$.

8. Identical-edge homogeneous networks. An identical-edge homogeneous cell network $\mathcal{G}$ is a homogeneous network in which all edges in $\mathcal{E}$ are equivalent.

Proposition 8.1. Every quotient of an identical-edge homogeneous network is an identicaledge homogeneous network.

Proof. This statement follows directly from section 5 (D) and Remark 5.1.

Proposition 8.2. Every identical-edge homogeneous network is the quotient of an identicaledge homogeneous network without multiple edges or self-coupling.

Proof. We begin by showing that if some cell in an $N$-cell network, say, cell 1 , has $m$ selfcouplings, then we can enlarge the network to an $(N+m)$-cell network, having the original network as a quotient, so that the enlarged network has no self-couplings in cells residing in the pullback of cell 1. Add arrows and cells to the enlarged network as follows.

(1) Replace cell 1 with $m+1$ input isomorphic cells. 
(2) For each arrow in the original network with head cell 1 and tail cell $i$, where $i \neq 1$, add $m$ edge-equivalent arrows with tail cell $i$, where one of the new arrows terminates in each of the $m$ new cells.

(3) Each pair of the $m+1$ cells in the preimage of cell 1 has identical arrows with head in the first cell of the pair and tail in the second cell of the pair.

Note that all arrows starting from one of the $m$ new cells terminate in cell 1 . In particular, none of the $m+1$ cells in the preimage of cell 1 have self-coupling arrows. In the new network, assign all cells in the preimage of cell 1 the same color and all other cells different colors. This coloring is balanced and yields the original network as a quotient network. See Figure 9. Therefore, we can enlarge the original network so that it has no self-coupling arrows.
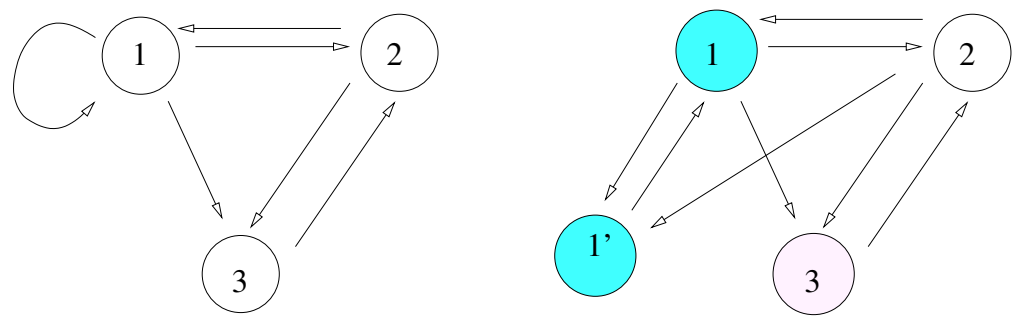

Figure 9. (Left) Three-cell network with self-coupling. (Right) Four-cell enlargement of original system.

Next, we assume that the network has no self-coupling and that there are $m$ identical arrows from cell 1 to cell 2 . There is an extended coupled cell network with $N+m-1$ cells formed by replacing cell 1 with $m$ identical cells and changing arrows as follows:

(1) Each of the $m$ cells replacing cell 1 connects to cell 2 with one arrow. Note that cell 2 receives the same number of arrows from the $m$ copies of cell 1 that it received previously from the single cell 1 in the original network.

(2) Add arrows so that every cell that was connected to cell 1 in the original network is now connected to each of the $m$ cell 1's in the new network.

Note that there are no arrows starting from one of the $m-1$ new cells that terminate in a cell in the original network not equal to cell 2. In the new network, color all cells in the preimage of cell 1 the same color and all other cells different colors. This coloring is balanced and yields the original network as a quotient network. See Figure 10. Proceeding inductively, we can eliminate multiple arrows between cells.
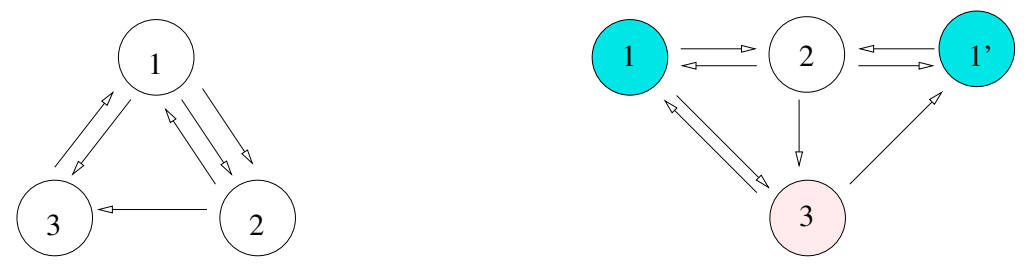

Figure 10. (Left) Three-cell network with multiple arrows. (Right) Four-cell enlargement of original system.

9. Hopf bifurcation in two-color networks. We now specialize our results to equivalence relations with two colors. We prove a Hopf bifurcation theorem in the case of an identical- 
edge homogeneous network, with the feature that well-defined approximate phase shifts and approximate amplitude relations hold near bifurcation.

Suppose that an identical-edge homogeneous network has a balanced relation with two colors. The corresponding quotient network has the form given in Figure 11. Indeed, Proposition 8.1 implies that the two cells are input isomorphic and all edges are identical. Such two-cell networks are determined by the number of self-coupling arrows $l_{j}$ on cell $j$ and the number of edges $m_{1} \geq 0$ coupling cell 2 to cell 1 . Let $m_{2} \geq 0$ be the number of edges coupling cell 1 to cell 2 ; then homogeneity implies $l_{1}+m_{1}=l_{2}+m_{2}$.

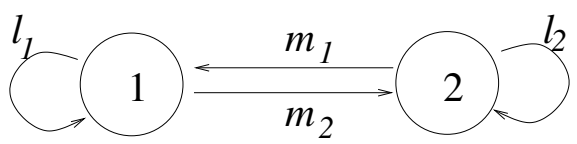

Figure 11. The two-cell quotient network.

Wang and Golubitsky [11] use this quotient network (with multiple arrows and selfcoupling) to prove that equilibria corresponding to balanced two-colorings may be obtained from a codimension-one steady-state bifurcation from a homogeneous equilibrium; we use the quotient to study Hopf bifurcations.

Proposition 9.1. Suppose that an identical-edge homogeneous network has a balanced relation with two colors and that the quotient network is not feed-forward, that is, $m_{1}, m_{2}>0$. Then there is a unique type of synchrony-breaking Hopf bifurcation from a synchronous equilibrium that leads to periodic solutions that are synchronous on all cells of the same color and that are approximately one half a period out of phase with all cells of the opposite color. The amplitudes of these periodic signals need not be equal.

Proof. The coupled cell systems have the form

$$
\begin{aligned}
& \dot{x}_{1}=f(x_{1}, \underbrace{x_{1}, \ldots, x_{1}}_{l_{1} \text { times }}, \underbrace{x_{2}, \ldots, x_{2}}_{m_{1} \text { times }}), \\
& \dot{x}_{2}=f(x_{2}, \underbrace{x_{2}, \ldots, x_{2}}_{l_{2} \text { times }}, \underbrace{x_{1}, \ldots, x_{1}}_{m_{2} \text { times }}),
\end{aligned}
$$

where $x_{1}, x_{2} \in \mathbf{R}^{k}$. Since $\left\{x: x_{1}=x_{2}\right\}$ is flow-invariant, we can arrange for the robust existence of an equilibrium in this subspace. Moreover, by a change of coordinates, we can assume that the equilibrium is at the origin. Let $J$ be the Jacobian matrix of this equilibrium. By (9.1)

$$
J=\left[\begin{array}{cc}
A+l_{1} B & m_{1} B \\
m_{2} B & A+l_{2} B
\end{array}\right]
$$

where $A$ is the linearization of the internal dynamics and $B$ is the coupling matrix. Assume that $x_{1}, x_{2} \in \mathbf{R}^{k}$. Let $v \in \mathbf{R}^{k}$ and observe that

$$
J\left[\begin{array}{l}
v \\
v
\end{array}\right]=\left[\begin{array}{c}
(A+p B) v \\
(A+p B) v
\end{array}\right] \quad \text { and } \quad J\left[\begin{array}{c}
m_{1} v \\
-m_{2} v
\end{array}\right]=\left[\begin{array}{c}
\left(A+\left(l_{2}-m_{1}\right) B\right) m_{1} v \\
-\left(A+\left(l_{2}-m_{1}\right) B\right) m_{2} v
\end{array}\right],
$$



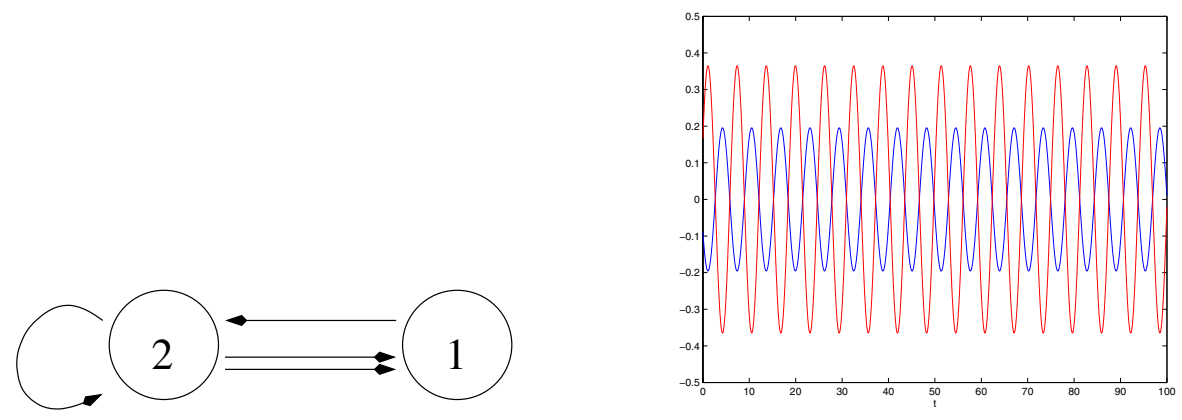

Figure 12. (Left) Two-cell homogeneous network. (Right) Half-period out of phase periodic state with different amplitudes obtained by Hopf bifurcation.

where $p=m_{1}+l_{1}=m_{2}+l_{2}$. Thus, the eigenvalues of $J$ are given by eigenvalues of the $k \times k$ matrices $A+p B$ and $A+\left(l_{2}-m_{1}\right) B$. Either matrix can have purely imaginary eigenvalues when $k \geq 2$. Critical eigenvalues in the matrix $A+p B$ lead to periodic solutions that are synchronous on all cells, since the synchrony subspace $x_{1}=x_{2}$ is flow-invariant.

Synchrony-breaking Hopf bifurcations occur if the matrix $A+\left(l_{2}-m_{1}\right) B$ has (simple) purely imaginary eigenvalues $\pm \omega i$. Let $v_{0} \in \mathbf{C}^{k}$ be an eigenvector associated to the eigenvalue $w i$. Then Hopf bifurcation can lead to a branch of periodic solutions that to first order in the bifurcation parameter has the form

$$
x_{1}(t)=m_{1} \operatorname{Re}\left(e^{i \omega t} v_{0}\right), \quad x_{2}(t)=-m_{2} \operatorname{Re}\left(e^{i \omega t} v_{0}\right) .
$$

The amplitudes of the time series $x_{1}(t)$ and $x_{2}(t)$ are different (unless $m_{1}=m_{2}$ ). Indeed, to first order they are in the ratio $m_{1}: m_{2}$ near the bifurcation point. The minus sign in $x_{2}$ shows that the time series are (to first order) a half-period out of phase.

Example 9.2. Consider the two-cell system in Figure 12 (left). This network can be obtained as a two-color quotient network of the five-cell network in Figure 1 (right) by identifying the four pink and white cells as one color and the cyan cell as the other color. The time series of a periodic state obtained by Hopf bifurcation in this network is shown in Figure 12 (right). Note that the time series from cells 1 and 2 are approximately one half a period out of phase even though the amplitudes of these signals are quite different. The amplitude ratio here is convincingly close to $m_{1} / m_{2}=2$. This coupled cell system has the form

$$
\begin{aligned}
& \dot{x}_{1}=f\left(x_{1}, x_{2}, x_{2}, \lambda\right), \\
& \dot{x}_{2}=f\left(x_{2}, x_{2}, x_{1}, \lambda\right) .
\end{aligned}
$$

The time series in Figure 12 (right) was obtained using $f: \mathbf{R}^{2} \times \mathbf{R}^{2} \times \mathbf{R}^{2} \rightarrow \mathbf{R}^{2}$, where

$$
f\left(y_{1}, y_{2}, y_{3}, \lambda\right)=\left(\left[\begin{array}{cc}
0 & -1 \\
1 & 0
\end{array}\right]+(\lambda-1) I_{2}\right) y_{1}-\left(y_{2}+y_{3}\right)-\left|y_{1}\right|^{2} y_{1}-\left(y_{2}, y_{3}\right) y_{1} .
$$

A supercritical Hopf bifurcation from the trivial equilibrium at the origin occurs at $\lambda=0$. In the given time series $\lambda=0.1$.

When $m_{1}=m_{2}\left(\right.$ so $\left.l_{1}=l_{2}\right)$ we can say more. 
Corollary 9.3. Suppose that an identical-edge homogeneous network has a balanced equivalence relation with two colors, black and white. If the number of white cells coupled to a white cell is equal to the number of black cells coupled to a black cell, then the synchrony-breaking Hopf bifurcation in Proposition 9.1 leads to robust periodic solutions that are synchronous on cells of the same color and exactly one half a period out of phase with cells of the opposite color.

Proof. When $m_{1}=m_{2}$ (and hence $\left.l_{1}=l_{2}\right)$ in Proposition 9.1, the transposition $\left(x_{1}, x_{2}\right) \mapsto$ $\left(x_{2}, x_{1}\right)$ is a symmetry of (9.1) and the bifurcating states have an exact spatio-temporal symmetry $x_{2}(t)=x\left(t+\frac{T}{2}\right)$, where $T$ is the (minimal) period.

10. Concluding remarks. Primary goals of our research are the study of the types of typical states that can occur in networks of coupled systems of differential equations (synchronous states are one of these) and the study of typical (codimension-one) synchrony-breaking bifurcations. Any study of genericity must begin with a precise description of the classes of differential equations that are to be considered, and any abstract study of synchrony-breaking bifurcations must begin with a definition of what synchrony means. In this paper and [10] we have set up a framework (based on groupoids) that specifies both the classes of differential equations (associated to a fixed network) and the notion of (robust) synchrony that can occur in that network (balanced relations).

An important observation is that the restrictions of coupled cell systems to polysynchronous subspaces are themselves coupled cell systems associated to the quotient network. This restriction has profound and interesting consequences for the generic behavior of polysynchronous dynamics. In this paper we have refined the notion of quotient dynamical systems, through the network theoretic conventions of the multiarrow formalism, to the point that genericity arguments on quotient networks now lift to genericity arguments about polysynchronous dynamics in the original network.

It is a nontrivial task to compute all balanced relations in a complicated network; nevertheless this is a simpler task than that of computing all flow-invariant subspaces for admissible vector fields. In certain instances, such as with certain types of colorings [7, 11], this classification can be completed, and these classifications provide interesting information about patterns of synchrony. The work in [7,9] shows that codimension-one synchrony-breaking bifurcations can be highly nonstandard (and complicated to analyze). A complete theory for synchrony-breaking will require a better understanding of the Jacobian matrices at synchronous equilibria (parallel to the representation theory of matrices commuting with a given group action). At present such a theory does not exist, but some initial steps can be found in [3].

Acknowledgments. We wish to thank Ana Dias and the referees for helpful discussions and suggestions.

\section{REFERENCES}

[1] H. Brandt, Über eine Verallgemeinerung des Gruppenbegriffes, Math. Ann., 96 (1927), pp. 360-366.

[2] R. Brown, From groups to groupoids: A brief survey, Bull. London Math. Soc., 19 (1987), pp. $113-134$. 
[3] A. P. S. DiAs AND I. STEWART, Symmetry groupoids and admissible vector fields for coupled cell networks, J. London Math. Soc. (2), 69 (2004), pp. 707-736.

[4] A. P. S. DiAs AND I. StewArT, Linear Equivalence and ODE-Equivalence for Coupled Cell Networks, submitted.

[5] I. R. Epstein And M. Golubitsky, Symmetric patterns in linear arrays of coupled cells, Chaos, 3 (1993), pp. $1-5$.

[6] D. Gillis And M. Golubitsky, Patterns in square arrays of coupled cells, J. Math. Anal. Appl., 208 (1997), pp. 487-509.

[7] M. Golubitsky, M. Nicol, And I. Stewhrt, Some curious phenomena in coupled cell networks, J. Nonlinear Sci., 14 (2004), pp. 119-236.

[8] P. J. Higgins, Notes on Categories and Groupoids, Van Nostrand Reinhold Mathematical Studies 32, Van Nostrand Reinhold, London, 1971.

[9] M. Leite AND M. Golubitsky, Synchrony-breaking bifurcations in homogeneous three-cell networks, in preparation.

[10] I. Stewart, M. Golubitsky, And M. Pivato, Symmetry groupoids and patterns of synchrony in coupled cell networks, SIAM J. Appl. Dynam. Sys., 2 (2003), pp. 609-646.

[11] Y. WANG AND M. Golubitsky, Two-color patterns of synchrony in lattice dynamical systems, Nonlinearity, 18 (2005), pp. 631-657.

[12] N. J. Wildberger, A new look at multisets, preprint, University of New South Wales, Sydney, 2003. 